
\title{
R Reserach S Surare \\ The Predictive Value of the Peripheral Blood Lymphocyte-to-Monocyte Ratio in Patients with Anaplastic Large Cell Lymphoma
}

\section{Shengnan Zhang}

Affiliated Tumor Hospital of Zhengzhou University: Henan Cancer Hospital https://orcid.org/00000001-5001-5276

\section{Yuqing Chen}

Henan Provincial People's Hospital

\section{Qian Wang}

Henan Cancer Hospital

\section{Fangfang Yuan}

Henan Cancer Hospital

\section{Hao Ai}

Henan Cancer Hospital

Lin Chen

Henan Cancer Hospital

\section{Ruihua Mi}

Henan Cancer Hospital

\section{Xudong Wei}

Henan Cancer Hospital

\section{Yongping Song}

Henan Cancer Hospital

Qingsong Yin ( $\nabla$ jnyinqingsong@163.com )

Henan Cancer Hospital

\section{Research}

Keywords: Anaplastic large cell lymphoma, Lymphocyte-to-monocyte ratio, ALK expression, Treatment response, Prognosis.

Posted Date: February 23rd, 2021

DOI: https://doi.org/10.21203/rs.3.rs-231724/v1 
License: (c) (i) This work is licensed under a Creative Commons Attribution 4.0 International License. Read Full License 


\section{Abstract}

\section{Background}

To assess the value of the peripheral blood lymphocyte-to-monocyte ratio (LMR) in predicting the early treatment response and prognosis in patients with anaplastic large cell lymphoma (ALCL).

\section{Methods}

The optimal cut-off value for the LMR was determined using the receiver operating characteristic curve (ROC) method. The LMR was monitored dynamically, and the relationship between the LMR and therapeutic response, and survival was analyzed.

\section{Results}

The cumulative complete remission (CCR) rate was $57.1 \%$ in patients with low baseline LMR, remarkably lower than that for patients with high baseline LMR (80.0\%) $(P=0.019)$. The 5-year overall survival (OS) and progression-free survival (PFS) were $51.3 \%$ and $32.6 \%$, respectively, in the low baseline LMR group, which were significantly poorer at $90.4 \%$ and $70.5 \%$ in the high baseline LMR group, respectively $(P$ values were 0.006 and 0.013 , respectively). Furthermore, the median OS and PFS of patients with a decreased LMR after treatment were shorter than those with an elevated LMR after treatment (all $P$ values $<0.05)$. Univariate and multivariate analysis demonstrated that low baseline LMR is an independent indicator for poor prognosis in ALCL patients ( $P$ values were 0.006 and 0.020 , respectively). Interestingly, the combination of low baseline LMR and ALK positive could strongly predict the good prognosis in patients with ALCL.

\section{Conclusions}

The low baseline LMR and a decreased LMR after treatment are prognostic indicator for poor PFS and $\mathrm{OS}$. The combination of LMR and ALK expression can widely risk-stratify patients for treatment and survival in patients with ALCL.

\section{Background}

Anaplastic large cell lymphoma (ALCL) is a common subtype of peripheral T-cell lymphoma (PTCL) that is characterized by clinical, pathological, and genetic heterogeneity, and it is divided as ALK positive and ALK negative by World Health Organization classification [1, 2]. At present, multiagent CHOP (cyclophosphamide, doxorubicin, vincristine and prednisone) or $\mathrm{CHOP}$-like regimens remain the standard of care for newly diagnosed ALCL patients. The role of consolidative high-dose therapy and autologous/allogeneic hematopoietic stem cell transplantation (HSCT) remains unclear. Multiple novel agents have recently been explored to improve the efficacy and prognosis of patients with ALCL, such as the anti-CD30 monoclonal antibody brentuximab vedotin, the ALK inhibitor crizotinib, and deacetylase inhibitors, particularly for relapsed or refractory $(R / R)$ patients [3-6]. 
Clinical characteristics (i.e., International Prognostic Index, IPI) generally predict survival in patients with ALCL [7]. In addition, ALK expression is an important prognostic marker; ALK-positive ALCL has remarkably better prognosis than ALK-negative ALCL or other peripheral T-cell lymphomas [8,9]. Recently, two recurrent chromosomal rearrangements involving DUSP22 and TP63 have been reported to predict survival as well, with the former being favorable and the latter an unfavorable prognostic marker [10]. However, there remains no accepted clinical prognostic index for ALCL patients. Early identification of high-risk patients is essential for clinical decision making; therefore, new prognosis markers need to be identified.

Chronic inflammation has been demonstrated to be associated with tumor development and progression $[11,12]$. Absolute lymphocyte counts (ALCs) in the peripheral blood reflect the host immune status [1316]. Absolute monocyte counts (AMCs) act as an indicator for tumor-associated macrophages [17, 18]. Recent studies have found that the lymphocyte-to-monocyte ratio (LMR) can be used as an inflammationrelated biomarker to assess immune function and the tumor microenvironment. A decreased LMR may reflect an innately dysfunctional host immune response, or a byproduct of tumor-related immune suppression [19]. Moreover, the low LMR is associated with the poor OS and PFS of patients, which has been confirmed in Hodgkin's lymphoma and a variety of non-Hodgkin's lymphomas, including diffuse large B-cell lymphoma (DLBCL), follicular lymphoma (FL), and NK/T-cell lymphoma [20-25]. However, the predictive value of the LMR has not been fully characterized in patients with ALCL.

Therefore, the purpose of this study is to evaluate the prognostic value of the peripheral blood LMR alone or combined with ALK expression in patients with ALCL.

\section{Methods}

\section{Patients selection}

This study is a retrospective analysis of a cohort of 103 newly diagnosed ALCL patients treated in the Affiliated Cancer Hospital and the Affiliated People's Hospital of Zhengzhou University between March 2012 and May 2019. The inclusion criteria were as follows: (1) a pathologically and immunohistochemically confirmed diagnosis of ALCL according to WHO 2016 classification of Tumors and Hematopoietic and Lymphoid Tissues [26] that was validated by more than two experienced professors from the Department of Pathology and (2) sufficient data during treatment and follow-up. Patients were excluded from this study if they had a history of anti-tumor treatment and clinical evidence of acute infection or chronic acute inflammatory disease.

Data collected included Eastern Cooperative Oncology Group (ECOG), performance status (PS), age, gender, involved sites, B symptoms, serum LDH level, serum $\beta 2 \mathrm{MG}$, bone marrow involvement, complete blood count (CBC), ALC, AMC, liver and renal function, serum potassium, sodium, chloride and calcium levels, IPI score, Ann-Arbor stage, and ALK expression. This study was approved by the Medical Ethical Committee of the Affiliated Cancer Hospital and the Affiliated People's Hospital of Zhengzhou University. 
All data were obtained after informed consent from the recruited patients in accordance with the Declaration of Helsinki.

\section{Measurement of the absolute lymphocyte/monocyte count and calculation of the LMR and LMR/LDH}

$\mathrm{CBC}$ and serum LDH levels were measured and recorded at various time points (at diagnosis, before each chemotherapy cycle, and during follow-up). The LMR was calculated as ALCs divided by AMCs. Similarly, LMR/LDH was calculated as the LMR value divided by the serum LDH value. The LMR and LMR/LDH at diagnosis (baseline LMR and LMR/LDH) were obtained within a week before the first course of chemotherapy, the LMR during treatment was before each chemotherapy treatment, and the LMR during follow-up was obtained every three months in the first year, every six months in the second year, and then once yearly. The optimal cut-off values for both the LMR and LMR/LDH were determined using Cutoff Finder via the ROC method (Euclidean distance).

\section{Regimens and responses}

All patients received CHOP-E (etoposide, prednisone, vincristine, cyclophosphamide, doxorubicin) or CHOP-like regimens for 6-8 cycles of chemotherapy as first-line treatment. Subsequently, autologous stem cell transplantation or chidamide was administered as consolidation, or salvage therapy. Among the 103 patients, 17 (16.5\%) underwent autologous stem cell transplantation. A total of $17(16.5 \%)$ patients were treated with chidamide, of which $6(5.8 \%)$ received chidamide combined with chemotherapy as firstline treatment, 8 (7.8\%) as second-line treatment, and $3(2.9 \%)$ received chidamide alone as maintenance treatment. The chemotherapy regimens are shown in Table 1. The proportions of patients treated with each regimen were balanced for the number of patients in the high and low LMR groups.

Overall survival (OS) was defined as the duration from disease diagnosis to final follow-up or death. Progression-free survival (PFS) was defined as the duration from disease diagnosis to disease progression or disease-related death. The responses were evaluated every two courses of chemotherapy, according to standard response criteria for $\mathrm{NHL}$ [27], the responses were divided as complete remission (CR), $C R u(C R$, unidentified), partial remission (PR), stable disease (SD), and progressive disease (PD), and $(\mathrm{CR}+\mathrm{CRu}+\mathrm{PR})$ served as the overall response rate (ORR).

In the follow-up analysis, $\mathrm{CBC}$, blood chemistry, and imaging data were obtained every three months in the first year, every six months in the second year, and then once yearly. The follow-up deadline was July 2019. The survival time of patients who were lost to follow up was calculated to the last follow-up date.

\section{Statistical analysis}


Categorical characteristics and ratios were compared using the Chi-square or Fisher's exact test. The Kaplan-Meier method was used to calculate the probability of survival, and differences between groups were compared with the log-rank test. A Cox proportional hazards regression model was applied to perform univariate and multivariate analysis. A two-sided $P<0.05$ was defined as significant. All statistical analyses were conducted using SPSS 21.0 and GraphPad Prism 7.0 software.

\section{Results}

\section{Characteristics of patients}

A total of 103 newly diagnosed ALCL patients with a median age of 31 years (range: 5-68 years) were eligible to be evaluated. Ninety-three (90.3\%) patients were $\leq 60$ years, the gender ratio was 1.5:1 (male: female), and ALK expression ratio was 68:35 (positive: negative). The median follow-up time was 33 months (range: 1-84 months). Twenty-eight patients (27.2\%) experienced recurrence or disease progression, and 14 patients (13.6\%) died. The median for ALCs was $1.5 \times 10^{9} / \mathrm{L}$ (range: $0.54-4.20$ ), and the median for AMCs was $0.5 \times 10^{9} / \mathrm{L}$ (range: 0.04-1.29). The patient characteristics are shown in Table 1 .

\section{Optimal cut-off values for the LMR and LMR/LDH}

The optimal cut-off value for the baseline LMR was 2.4, and the area under the curve (AUC) was 0.687 (95\% Cl: $0.532-0.843 ; P=0.025 ;$ Fig. 1 A). A total of 75 patients $(72.8 \%)$ with $L M R>2.4$ were defined as the high baseline LMR group, and 28 patients $(27.2 \%)$ with LMR $\leq 2.4$ were defined as the low baseline LMR group. The optimal cut-off value for LMR/LDH was 1.4, and the AUC was 0.738 (95\% Cl: 0.625 $0.851 ; P=0.004$; Fig. 1B). A total of 57 patients (55.3\%) had an LMR/LDH > 1.4, and 46 patients (44.7\%) had an LMR/LDH $\leq 1.4$.

\section{The low baseline LMR Is significantly associated with high IPI score, B symptom, increased $\beta 2-\mathrm{MG}$, and Ann-Arbor stage $0-\square$.}

Several clinical factors were associated with the low baseline LMR in ALCL patients (Table 1). Specifically, low baseline LMR was common in high-risk patients with an IPI score 3-5 (56.5\%), while it was rare in patients with IPI score $0-2(18.8 \%)(P<0.001)$. Similarly, low baseline LMR was more frequent in patients with $B$ symptoms (35.8\%) than in patients without B symptoms $(18.0 \%)(P=0.042)$. And low baseline LMR was significantly related to increased serum $\beta 2-M G(P=0.036)$. Additionally, compared with $12.9 \%$ of patients with Ann-Arbor stage $\mathbb{Z}-\mathbb{\bigotimes}, 33.3 \%$ of advanced patients with Ann-Arbor stage $\mathbb{X}-\mathbb{Z}$ had an LMR $\leq 2.4(P=0.033)$. 


\section{The baseline LMR can predict early response to treatment and prognosis for ALCL patients}

The CCR rate of the low baseline LMR group (57.1\%) was remarkably lower than that for the high baseline LMR group $(80.0 \%, P=0.019)$. When a good response (CR+PR) was obtained in the high LMR group, the LMR was decreased significantly $(P=0.030)$ but not for patients with a poor response $(P=$ 0.086). No significant difference was found in the low LMR group regardless of whether a response was achieved or not ( $P$ values were 0.137 and 0.358 ).

The Low baseline LMR was significantly related to poor OS (Fig. 2A, $P=0.006$ ) and PFS (Fig. 2B, $P=$ 0.013), and the 5-year OS and PFS were $51.3 \%$ and $32.6 \%$, respectively, in the low LMR group, and $90.4 \%$ and $70.5 \%$, respectively, in the high LMR group. Meanwhile, low baseline LMR/LDH was significantly associated with shorter OS (Fig. 2C, $P=0.002$ ) and PFS (Fig. 2D, $P=0.029$ ) when compared with that of the high baseline LMR group, and the 5-year OS and PFS were $57.4 \%$ and $52.3 \%$, respectively, in the low LMR/LDH group, and $92.8 \%$ and $74.9 \%$, respectively, in the high LMR/LDH group.

\section{The baseline LMR is highly consistent with ALK expression in predicting prognosis of patients with ALCL}

In this study, we found that the CCR rate in the ALK-negative group (57.1\%) was significantly lower than that in the ALK-positive group (82.4\%) $(P=0.006)$. The OS (Fig. 3A, $P=0.005)$ and PFS (Fig. 3B, $P=$ 0.012) in the ALK-negative group were significantly poorer than that in the ALK-positive group, and the 5year OS and PFS were $60.4 \%$ and $47.1 \%$, respectively, in the ALK-negative group, and $92.8 \%$ and $76.2 \%$, respectively, in the ALK-positive group. Although the baseline LMR and ALK expression are highly consistent in terms of predicting efficacy and prognosis, there was no significant correlation between the two $(R=0.10)$.

To compare the prognostic power of the baseline LMR, ALK expression, and their combination for ALCL patients, we divided the 103 cases into four subgroups: Group a: patients who were ALK positive and had a baseline LMR > 2.4, Group b: patients who were ALK positive and had a baseline LMR $\leq 2.4$, Group c: patients who were ALK negative with a baseline LMR > 2.4, and Group d: patients who were ALK negative with a baseline $L M R \leq 2.4$. We found that a baseline $L M R>2.4$ combined with ALK positive were significantly associated with better OS (Fig. 3C, $P<0.001$ ) and PFS (Fig. 3D, $P=0.002$ ) when compared with the other three groups. In contrast, patients who were ALK negative with a baseline LMR $\leq 2.4$ had the worst prognosis compared to patients who were ALK positive and/or those with a baseline LMR > 2.4 .

\section{The survival of patients with an increased LMR after treatment is greater than that for patients with a decreased}




\section{LMR after treatment.}

To observe changes in LMR during treatment and their effect on prognosis, the LMR at various time points during treatment and the follow-up was dynamically monitored, and the patients were divided into two subgroups: Group 1, those with an increased LMR at the end of therapy (greater than or equal to the baseline LMR), and Group 2, those with a decreased LMR after treatment (less than the baseline LMR). It was found that patients in Group 1 had a longer OS (Fig. 4A, $P=0.014$ ) and PFS (Fig. 4B, $P=0.018$ ) than those in Group 2, and the 5-year OS and PFS were $92.05 \%$ and $75.88 \%$, respectively, in Group 1, and $69.48 \%$ and $57.68 \%$, respectively, in Group 2. Additionally, we found that different chemotherapy regimens had no effect on the LMR (Suppl Fig. 1); nevertheless, at 6-9 months of the follow-up, the LMR of patients treated with chidamine was significantly lower than that for patients not treated with chidamine until 12 months later (Fig. 5A, $P=0.046$ at 6 months and 0.037 at 9 months, respectively). As expected, compared with chemotherapy, chidamine combined with chemotherapy demonstrated a mild advantage in PFS and OS for both newly diagnosed (Fig. 5B and Fig. 5C) and R/R patients (Fig. 5D and 5E), but there was no statistically significant difference (All $P$ values $>0.05$ ).

In addition, the LMR at recurrence was lower than that at last follow-up in the high and low LMR groups, but there was no statistically significant difference (Suppl Fig. 2, $P=0.082$ and 0.317 , respectively).

\section{Survival analysis and prognosis factors}

Univariate analysis is shown in Table 2. ECOG PS $\geq 2$, Ann-Arbor stage $\geq \mathbb{\Downarrow}$, ALK negative, IPI Score $\geq 3$, $A L C \leq 1.5 \times 10^{9} / L, A M C>0.5 \times 10^{9} / L$, elevated serum LDH level, elevated serum $\beta 2-M G$, extranodal site involvement, LMR $\leq 2.4$, and LMR/LDH $\leq 1.4$ could be significantly associated with shortened OS and PFS. Clinical factors with statistical significance for OS and PFS $(P<0.05)$ were included in the multivariate analysis (Table 3). Multivariate analysis demonstrated that LMR was a poor prognostic factor for OS $(P=0.02)$ and PFS $(P=0.013)$.

\section{Discussion}

IPI score and ALK expression are widely used to predict the prognosis of ALCL patients, but they have their limitations $[7,8]$. In this retrospective study, we found for the first time that the low baseline LMR can be used as an indicator of poor prognosis and early efficacy in patients with ALCL. More specifically, the low baseline LMR had the potential to predict short OS and PFS, as well as poor early response to treatment. Furthermore, the patients with a decreased LMR after treatment had poor prognosis, with the exception of patients treated with the deacetylase inhibitor chidamide. The combination of baseline LMR $>2.4$ and ALK positive could strongly predict the good prognosis in patients with ALCL.

It is well known that ALK expression can predict early response to treatment and prognosis of ALCL patients. The CCR rate and survival of ALK-positive patients were significantly better than that for ALKnegative patients in this study, which is in line with the literature [8]. Recently, the baseline LMR has also 
been confirmed to have potential prognostic value for various malignant diseases [21-25]. Herein, we investigated the prognostic significance of baseline LMR independent or combined with ALK expression in a cohort of 103 ALCL patients. It was found that patients with a low baseline LMR had a higher CCR rate, and the 5-year OS and PFS of these patients were significantly poorer than that of patients with high baseline LMR, which is in accordance with a previous report [28]. In addition, we also found the low baseline LMR was significantly associated with several clinicopathologic factors, such as high IPI score, advanced disease, increased serum $\beta 2-\mathrm{MG}$, and B symptoms, which were commonly used to predict the poor prognosis of lymphoma patients [7]. More importantly, we observed that the baseline LMR was highly consistent with ALK expression, and multivariate analysis demonstrated that the two factors had the same weight in terms of its predictive value for ALCL patients. The combination of the above two factors has a more powerful prognostic effect than either single factor, especially for ALCL patients with better prognosis. These data suggest the baseline LMR can be used as an indicator for early response to treatment and prognosis of ALCL patients. Based on ALK negative and low baseline LMR, we can stratify patients at risk and give more intensive chemotherapy, targeted drugs, and even HSCT to high-risk patients.

The LMR level reflects a complex interaction between the tumor and the host immune response. We found the survival of patients with a further elevated LMR after treatment was significantly longer than that for patients with a decreased LMR, suggesting that patients with an elevated LMR after treatment have faster immune function reconstruction, are more likely to benefit from chemotherapy. By contrast, patients with a decreased LMR after treatment had poor prognosis, with the exception of patients treated with the deacetylase inhibitor chidamide. In addition, serum LDH is a general marker for predicting overall tumor load and cell turnover in patients with malignant neoplasms; thus, the LMR to LDH (LMR/LDH) ratio was a better predictor than the LMR alone for patients with NHL [29]. Here, we found that LMR/LDH highly coincided with the LMR alone in predicting the survival of patients with ALCL.

Chidamide has good efficacy and acceptable safety in patients with T-cell lymphoma [6]. As expected, compared with chemotherapy alone, chidamine combined with chemotherapy could improve the survival of patients with ALCL in this study, but there was no significant difference between the two treatments due to the small number of patients receiving chidamine. More interestingly, we also observed that chidamide could lead to a transient and significant decrease in the LMR after 6-9 months of treatment, which is contrary to the aforementioned point of view that the decrease in the LMR after treatment is associated with poor prognosis, and this may be related to the reduction in Treg cells induced by HDACis including chidamide [30,31]. In addition, previous studies have shown HDACis could preferentially induce the growth arrest and apoptosis of tumor cells and activate NK-mediated and CD8+ CTL-mediated adaptive anti-tumor immunity through enhanced CD8+ T cell and macrophage infiltration and decreased Treg cells [32,33], which may explain the above puzzling phenomena.

The LMR is an effective and simple indicator that can predict the prognosis and early efficacy of patients with ALCL, and can be readily obtained from a peripheral complete blood count. Therefore, it has been suggested that baseline LMR and its changes after treatment can be monitored dynamically to risk- 
stratify patients and guide treatment. However, there are some limitations to this study e.g., this was a two-center, retrospective study. Thus, it is necessary to conduct a multi-center and prospective study and to expand the sample size to verify these results.

\section{Conclusions}

This study found that the LMR can be used as an indicator of prognosis and early efficacy in patients with ALCL. The patients with a low baseline LMR and a decreased LMR after treatment had poor prognosis, and these patients may have a sufficient reason to change the current routine treatment. On the contrary, a high baseline LMR and an elevated LMR after treatment were strongly associated with good clinical outcomes, reflecting innate, residual immune function and rapid immune reconstitution in the host. Additionally, the combination of the high baseline LMR and ALK positive could strongly predict the good prognosis in patients with ALCL.

\section{Abbreviations}

ALCL: anaplastic large cell lymphoma; CCR: cumulative complete remission; LMR: lymphocyte to monocyte ratio; ALK: anaplastic lymphoma kinase; PR: partial remission; OS: overall survival; PFS: progression-free survival; ECOG: Eastern Cooperative Oncology Group; PS: performance status; SD: stable disease; PD: progressive disease; LDH: lactate dehydrogenase; $\beta 2$ MG: $\beta 2$ microglobulin; IPI: International Prognostic Index.

\section{Declarations}

Acknowledgments

Not applicable.

Authors' contributions

SNZ collected the patient information, analyzed the data, designed the figures, and wrote the manuscript. QW and HA collected data and performed statistical analysis. FFY, RHM, and LC collected clinical and literature information. YQC, XDW, and YPS participated in the analysis and interpretation of the data. QSY designed the study, analyzed the data, and wrote and revised the manuscript. All authors read and approved the final manuscript.

Funding

This work was supported by the Henan Provincial Medical Science and Technology Research Project (constructed by the Ministry of Education) (201701028) and the Natural Science Foundation of Henan Province (162300410280).

Availability of data and materials 
Data supporting the findings of this work are available within the paper and

its Additional Information files.

Ethics approval and consent to participate

The ethics committees of the Affiliated Cancer Hospital and the Affiliated People's Hospital of Zhengzhou University have approved this study.

Consent for publication

Not applicable.

Competing interests

The authors declare that they have no competing interests.

\section{References}

1. Irshaid $L, X u M L$. ALCL by any other name: the many facets of anaplastic large cell lymphoma. Pathology. 2020;52(1):100-110.

2. Tsuyama N, Sakamoto K, Sakata S, Dobashi A, Takeuchi K. Anaplastic large cell lymphoma: pathology, genetics, and clinical aspects. J Clin Exp Hematop. 2017;57(3):120-142.

3. Cederleuf $\mathrm{H}$, Bjerregård Pedersen $\mathrm{M}$, Jerkeman $\mathrm{M}$, Relander T, d'Amore F, Ellin $\mathrm{F}$. The addition of etoposide to CHOP is associated with improved outcome in ALK+ adult anaplastic large cell lymphoma: A Nordic Lymphoma Group study. Br J Haematol. 2017;178(5):739-746.

4. Locatelli F, Mauz-Koerholz C, Neville K, Llort A, Beishuizen A, Daw S, et al. Brentuximab vedotin for paediatric relapsed or refractory Hodgkin's lymphoma and anaplastic large-cell lymphoma: a multicentre, open-label, phase 1/2 study. Lancet Haematol. 2018;5(10):e450-461.

5. Foss F, Coiffier B, Horwitz S, Pro B, Prince HM, Sokol L, et al. Tolerability to romidepsin in patients with relapsed/refractory T-cell lymphoma. Biomark Res. 2014;8(2):16.

6. Shi Y, Jia B, Xu W, Li W, Liu T, Liu P, et al. Chidamide in relapsed or refractory peripheral T cell lymphoma: a multicenter real-world study in China. J Hematol Oncol. 2017;10(1):69.

7. Sonnen R, Schmidt WP, Müller-Hermelink HK, Schmitz N. The International Prognostic Index determines the outcome of patients with nodal mature T-cell lymphomas. $\mathrm{Br} \mathrm{J}$ Haematol. 2005;129(3):366-372.

8. Xing X, Feldman AL. Anaplastic large cell lymphomas: ALK positive, ALK negative, and primary cutaneous. Adv Anat Pathol. 2015;22(1):29-49.

9. Hapgood G, Savage KJ. The biology and management of systemic anaplastic large cell lymphoma. Blood. 2015;126(1):17-25. 
10. Vasmatzis G, Johnson SH, Knudson RA, Ketterling RP, Braggio E, Fonseca R, et al. Genome-wide analysis reveals recurrent structural abnormalities of TP63 and other p53-related genes in peripheral T-cell lymphomas. Blood. 2012;120(11):2280-2289.

11. Yu Y, Cui JW. Present and future of cancer immunotherapy: A tumor microenvironmental perspective. Oncol Lett. 2018;16(4):4105-4113.

12. Bilen MA, Martini DJ, Liu Y, Lewis C, Collins HH, Shabto JM, et al. The prognostic and predictive impact of inflammatory biomarkers in patients who have advanced-stage cancer treated with immunotherapy. Cancer. 2019;125(1):127-134.

13. Bartlett EK, Flynn JR, Panageas KS, Ferraro RA, Sta Cruz JM, Postow MA, et al. High neutrophil-tolymphocyte ratio (NLR) is associated with treatment failure and death in patients who have melanoma treated with PD-1 inhibitor monotherapy. Cancer. 2020;126(1):76-85.

14. Yang J, Zhang L, Yu C, Yang XF, Wang H. Monocyte and macrophage differentiation: circulation inflammatory monocyte as biomarker for inflammatory diseases. Biomark Res. 2014;2(1):1.

15. Qi Y, Liao D, Mei D, Zhang Y, Liu Y. Elevated neutrophil-to-lymphocyte ratio is associated with poor outcomes for melanoma patients treated with PD-1 inhibitor or chemotherapy in a Chinese population. Front Oncol. 2020;10:1752.

16. Zhang YP, Zhang R, Zhu HY, Wang L, Wu YJ, Liang JH, et al. Circulating Low Absolute CD4+ T Cell Counts May Predict Poor Prognosis in Extranodal NK/T-Cell Lymphoma Patients Treating with Pegaspargase-Based Chemotherapy. Cancer Res Treat. 2018;51(1):368-377.

17. Sawa-Wejksza K, Kandefer-Szerszeń M. Tumor-associated macrophages as target for antitumor therapy. Arch Immunol Ther Exp. 2018;66(2):97-111.

18. Yang J, Zhang L, Yu C, Yang XF, Wang H. Monocyte and macrophage differentiation: circulation inflammatory monocyte as biomarker for inflammatory diseases. Biomark Res. 2014;2(1):1.

19. Qi Q, Zhuang $L$, Shen $Y$, Geng $Y$, Yu S, Chen $H$, et al. A novel systemic inflammation response index (SIRI) for predicting the survival of patients with pancreatic cancer after chemotherapy. Cancer. 2016;122(14):2158-2167.

20. Mano Y, Yoshizumi T, Yugawa K, Ohira M, Motomura T, Toshima T, et al. Lymphocyte-to-Monocyte Ratio is a Predictor of Survival after Liver Transplantation for Hepatocellular Carcinoma. Liver Transpl. 2018;24(11):1603-1611.

21. Romano A, Parrinello NL, Vetro C, Chiarenza A, Cerchione $C$, Ippolito $M$, et al. Prognostic meaning of neutrophil to lymphocyte ratio (NLR) and lymphocyte to monocyte ratio (LMR) in newly diagnosed Hodgkin lymphoma patients treated upfront with a PET-2 based strategy. Ann Hematol. 2018;97(6):1009-1018.

22. Wang J, Gao K, Lei W, Dong L, Xuan Q, Feng M, et al. Lymphocyte-to-monocyte ratio is associated with prognosis of diffuse large B-cell lymphoma: correlation with $\mathrm{CD} 163$ positive $\mathrm{M} 2$ type tumorassociated macrophages, not PD-1 positive tumor-infiltrating lymphocytes. Oncotarget. 2016;8(3):5414-5425. 
23. Marcheselli L, Bari A, Anastasia A, Botto B, Puccini B, Dondi A, et al. Prognostic roles of absolute monocyte and absolute lymphocyte counts in patients with advanced-stage follicular lymphoma in the rituximab era: an analysis from the FOLL05 trial of the Fondazione Italiana Linfomi. Br J Haematol. 2015;169(4):544-551.

24. Zhang S, Li M, Yuan F, Chen L, Mi R, Wei X, et al. The predictive value of dynamic monitoring of peripheral blood lymphocyte to monocyte ratio in patients with extranodal NK/T cell lymphoma. Cancer Cell Int. 2019;19:272.

25. Zhang SN, Li MJ, Yuan FF, Chen L, Mi RH, Wei XD, et al. Efficacy and prognosis of the dynamic monitoring lymphocyte to monocyte ratio in patients with diffuse large B-cell lymphoma. Zhonghua Yi Xue Za Zhi. 2019;99(40):3139-3144.

26. Swerdlow SH, Campo E, Pileri SA, Harris NL, Stein H, Siebert R, et al. The 2016 revision of the World Health Organization classification of lymphoid neoplasms. Blood. 2016;127(20):2375-2390.

27. Cheson BD, Fisher RI, Barrington SF, Cavalli F, Lister TA. Recommendations for initial evaluation, staging, and response assessment of Hodgkin and non-Hodgkin lymphoma: the Lugano classification. J Clin Oncol. 2014;32(27):3059-3068.

28. Jia T, Zhang R, Zhu HY, Liang JH, Wang L, Wu W, et al. Prognostic significance of peripheral blood absolute monocyte count and lymphocyte to monocyte ratio in anaplastic large cell lymphoma. Cancer Biomark. 2018;22(4):807-813.

29. Ji H, Niu X, Yin L, Wang Y, Huang L, Xuan Q, et al. Ratio of Immune Response to Tumor Burden Predicts Survival Via Regulating Functions of Lymphocytes and Monocytes in Diffuse Large B-Cell Lymphoma. Cell Physiol Biochem. 2018;45(3):951-961.

30. Wang L, Beier UH, Akimova T, Dahiya S, Han R, Samanta A, et al. Histone/protein deacetylase inhibitor therapy for enhancement of Foxp3+ T-regulatory cell function posttransplantation. Am J Transplant. 2018;18(7):1596-1603.

31. Bae J, Hideshima T, Tai YT, Song Y, Richardson P, Raje N, et al. Histone deacetylase (HDAC) inhibitor ACY241 enhances anti-tumor activities of antigen-specific central memory cytotoxic $T$ lymphocytes against multiple myeloma and solid tumors. Leukemia. 2018;32(9):1932-1947.

32. Cassetta L, Pollard JW. Repolarizing macrophages improves breast cancer therapy. Cell Res. 2017;27(8):963-964.

33. Conte M, De Palma R, Altucci L. HDAC inhibitors as epigenetic regulators for cancer immunotherapy. Int J Biochem Cell Biol. 2018;98:65-74.

\section{Tables}

Table 1 Clinical Characteristics of 103 Patients By the LMR Level at Diagnosis 


\begin{tabular}{|c|c|c|c|c|}
\hline Characteristic & No.๑\%ם & $\begin{array}{l}\text { Low LMR group } \\
\text { LMR } \leq 2.4\end{array}$ & $\begin{array}{l}\text { High LMR group } \\
\text { LMR }>2.4\end{array}$ & $P$ \\
\hline Gender & & & & 0.155 \\
\hline male & $62(60.2)$ & $20(32.3)$ & 42(67.7) & - \\
\hline female & $41(39.8)$ & $8(19.5)$ & $33(80.5)$ & - \\
\hline Age】y[ & & & & 0.545 \\
\hline$>60$ & $10(9.7)$ & $3(30.0)$ & $7(70.0)$ & - \\
\hline$\leq 60$ & $93(90.3)$ & $25(26.9)$ & $68(73.1)$ & - \\
\hline B symptoms & & & & 0.042 \\
\hline absent & $50(48.5)$ & $9(18.0)$ & $41(82.0)$ & - \\
\hline present & $53(51.5)$ & $19(35.8)$ & $34(64.2)$ & - \\
\hline Ann-Arbor stage & & & & 0.033 \\
\hline$\nabla-\nabla$ & $31(30.1)$ & $4(12.9)$ & $27(87.1)$ & - \\
\hline$\nabla-\nabla$ & 72(69.9) & $24(33.3)$ & $48(66.7)$ & - \\
\hline ECOG PS & & & & 0.357 \\
\hline$<2$ & 95(92.2) & $24(25.3)$ & $71(74.7)$ & - \\
\hline$\geq 2$ & $8(7.8)$ & $4(50.0)$ & $4(50.0)$ & - \\
\hline Serum LDH & & & & 0.085 \\
\hline$\leq$ normal & $72(69.9)$ & $16(22.2)$ & $56(77.8)$ & - \\
\hline$>$ normal & $31(30.1)$ & $12(38.7)$ & $19(61.4)$ & - \\
\hline Serum $\beta 2-M G$ & & & & 0.036 \\
\hline$\leq$ normal & $68(66.0)$ & $14(20.6)$ & $54(79.4)$ & - \\
\hline$>$ normal & $35(34.0)$ & $14(40.0)$ & $21(60.0)$ & - \\
\hline IPI score & & & & $<0.001$ \\
\hline $0-2$ & $80(77.7)$ & $15(18.8)$ & $65(81.2)$ & - \\
\hline $3-5$ & $23(22.3)$ & $13(56.5)$ & $10(43.5)$ & - \\
\hline Extranodal sites involvement & & & & 0.363 \\
\hline Yes & $55(53.4)$ & $17(30.9)$ & $38(69.1)$ & - \\
\hline No & $48(46.6)$ & $11(22.9)$ & $37(77.1)$ & - \\
\hline
\end{tabular}




\begin{tabular}{|lllll|}
\hline ALK expression & & & & 0.810 \\
\hline Positive & $68(66.0)$ & $19(27.9)$ & $49(72.1)$ & - \\
\hline Treatment & $35(34.0)$ & $9(25.7)$ & $26(74.3)$ & - \\
\hline CHOP-E & & & & 0.756 \\
EPOCH & $57(55.3)$ & $17(29.8)$ & $40(70.2)$ & - \\
\hline Hyper-CVAD & $20(19.4)$ & $6(30.0)$ & $14(70.0)$ & - \\
\hline BMF-NHL & $12(11.7)$ & $2(16.7)$ & $10(83.3)$ & - \\
\hline Chidamide & $14(13.6)$ & $3(21.4)$ & $11(78.6)$ & - \\
\hline Yes & & & & 0.293 \\
\hline No & $17(16.5)$ & $6(35.3)$ & $11(64.7)$ & - \\
\hline Stem cell transplantation & $86(83.5)$ & $22(25.6)$ & $64(74.4)$ & - \\
\hline
\end{tabular}

Table 2 Univariate Analysis of Risk Factors Associated With OS and PFS

\begin{tabular}{|lllll|}
\hline Yes & $17(16.5)$ & $7(41.2)$ & $10(58.8)$ & - \\
\hline No & $86(83.5)$ & $21(24.4)$ & $65(75.6)$ & - \\
\hline Bone marrow involvement & & & & 0.703 \\
Positive & $4(3.9)$ & $1(25.0)$ & $3(75.0)$ & - \\
\hline Negative & $99(96.1)$ & $27(27.3)$ & $72(72.7)$ & - \\
\hline
\end{tabular}

Abbreviations:CHOP-E, etoposide, prednisone, vincristine, cyclophosphamide, doxorubicin); EPOCH, etoposide, prednisone, vincristine, cyclophosphamide, doxorubicin; Hyper-CVAD, cyclophosphamide, vincristine, doxorubicin, dexamethasone,

Table 3 Multivariate Analysis of Risk Factors Associated With OS and PFS 


\begin{tabular}{|c|c|c|c|c|}
\hline Variables & os & & PFS & \\
\hline & $\mathrm{HR}(95 \% \mathrm{Cl})$ & $P$ value & $\mathrm{HR}(95 \% \mathrm{Cl})$ & $P$ value \\
\hline $\begin{array}{l}\text { Age } \\
(>60 \text { vs. } \leq 60)\end{array}$ & $0.57(0.09-3.42)$ & 0.540 & $1.41(0.41-4.81)$ & 0.586 \\
\hline $\begin{array}{l}\text { Gender } \\
\text { (male vs. female) }\end{array}$ & $0.56(0.19-1.62)$ & 0.320 & $1.23(0.58-2.61)$ & 0.581 \\
\hline $\begin{array}{l}\text { B symptoms } \\
\text { (yes vs. no) }\end{array}$ & $1.02(0.36-2.91)$ & 0.975 & $0.61(0.29-1.29)$ & 0.195 \\
\hline $\begin{array}{l}\text { ECOG PS } \\
(\geq 2 v s .<2)\end{array}$ & $3.39(0.98-11.69)$ & $<0.001$ & $1.31(0.51-3.32)$ & 0.574 \\
\hline 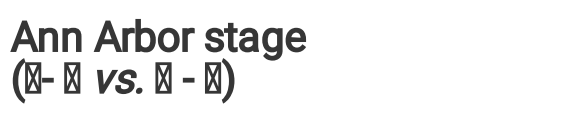 & $3.26(1.07-9.94)$ & 0.038 & $2.96(1.35-6.47)$ & 0.006 \\
\hline $\begin{array}{l}\text { ALK expression } \\
\text { (positive vs. negative) }\end{array}$ & $4.50(1.51-13.44)$ & 0.005 & $2.80(1.25-6.25)$ & 0.012 \\
\hline $\begin{array}{l}\text { IPI Score } \\
(0-2 \text { vs. 3-5) }\end{array}$ & $5.10(1.87-13.86)$ & $<0.001$ & $2.17(1.08-4.35)$ & 0.030 \\
\hline $\begin{array}{l}\text { Serum LDH } \\
\text { (normal vs. elevated) }\end{array}$ & $3.41(1.06-10.93)$ & 0.016 & $1.96(1.07-3.57)$ & 0.028 \\
\hline $\begin{array}{l}\text { Serum } \beta 2-M G \\
\text { (normal vs. elevated) }\end{array}$ & $5.22(2.36-11.51)$ & $<0.001$ & $2.31(1.05-5.08)$ & 0.022 \\
\hline $\begin{array}{l}\text { Extranodal sites involvement } \\
\text { (yes vs. no) }\end{array}$ & $3.13(1.38-7.09)$ & 0.006 & $1.85(1.04-3.28)$ & 0.037 \\
\hline $\begin{array}{l}\text { Bone marrow involvement } \\
\text { (yes vs. no) }\end{array}$ & $0.158(0.01-1.67)$ & 0.119 & $0.26(0.02-3.76)$ & 0.045 \\
\hline $\begin{array}{l}\text { ALC } \\
\left(\leq 1.5 \times 10^{9} / \mathrm{L} v s .>1.5 \times 10^{9} / \mathrm{L}\right)\end{array}$ & $4.22(1.43-12.46)$ & 0.009 & $2.34(1.09-5.34)$ & 0.018 \\
\hline $\begin{array}{l}\text { AMC } \\
\left(\leq 0.5 \times 10^{9} / L \text { vs. }>0.5 \times 10^{9} / L\right)\end{array}$ & $2.45(1.14-5.27)$ & 0.021 & $1.64(0.77-3.48)$ & 0.043 \\
\hline $\begin{array}{l}\operatorname{LMR} \\
(\leq 2.4 \text { vs. }>2.4)\end{array}$ & $4.36(1.27-15.02)$ & 0.006 & $2.17(1.12-4.10)$ & 0.013 \\
\hline $\begin{array}{l}\text { LMR/LDH } \\
(\leq 1.4 \text { vs. }>1.4)\end{array}$ & $4.81(1.65-14.04)$ & 0.002 & $2.05(0.96-4.39)$ & 0.029 \\
\hline
\end{tabular}


Figures
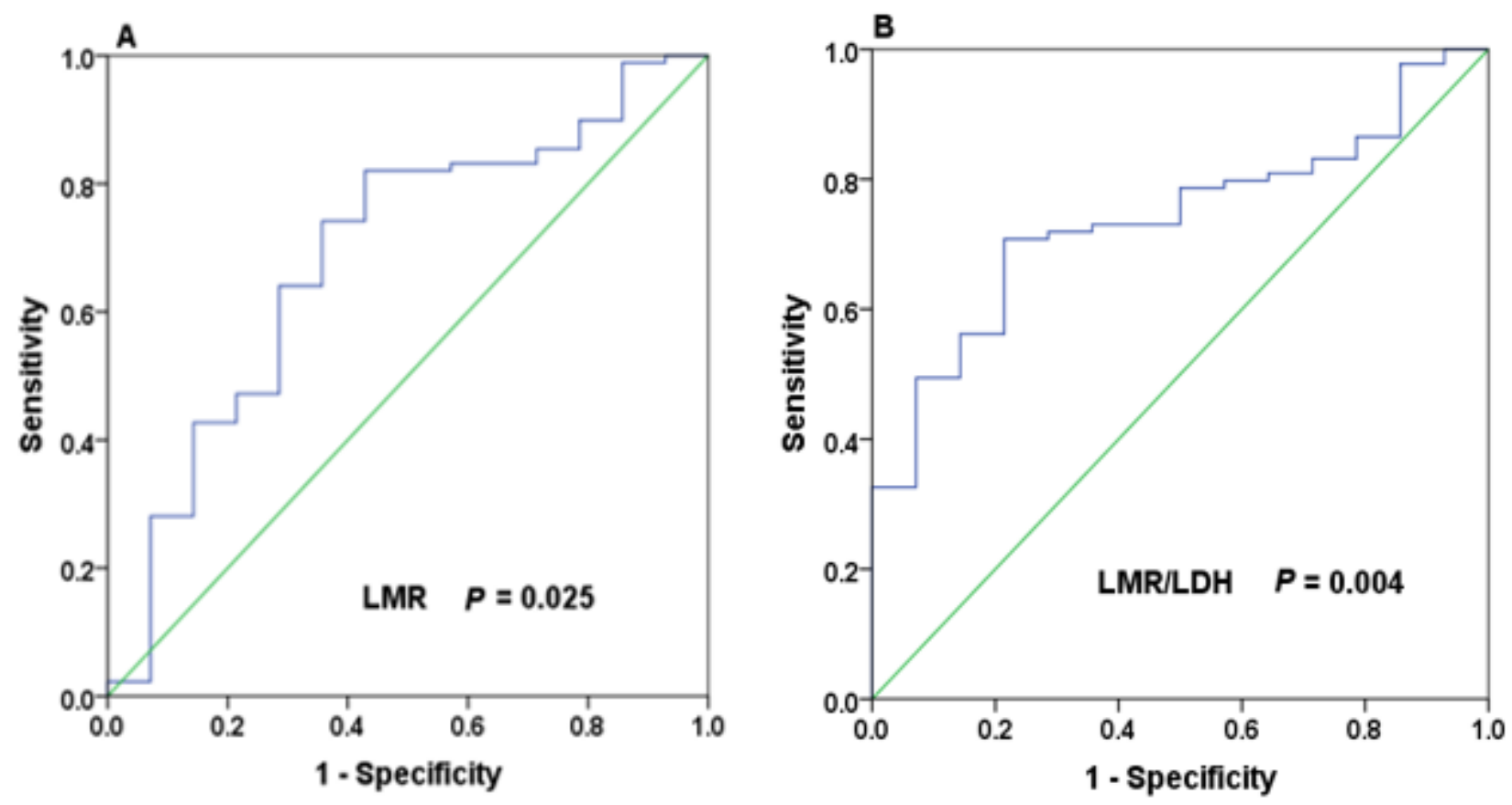

Figure 1

ROC curve analysis of the baseline LMR and LMR/LDH. A The optimal cut-off value for the baseline LMR was 2.40 (AUC: $0.687,95 \% \mathrm{Cl}: 0.532-0.843, \mathrm{P}=0.025$ ). B The optimal cut-off value for baseline LMR/LDH was 1.40 (AUC: $0.738,95 \% \mathrm{Cl}: 0.625-0.851, \mathrm{P}=0.004$ ). 

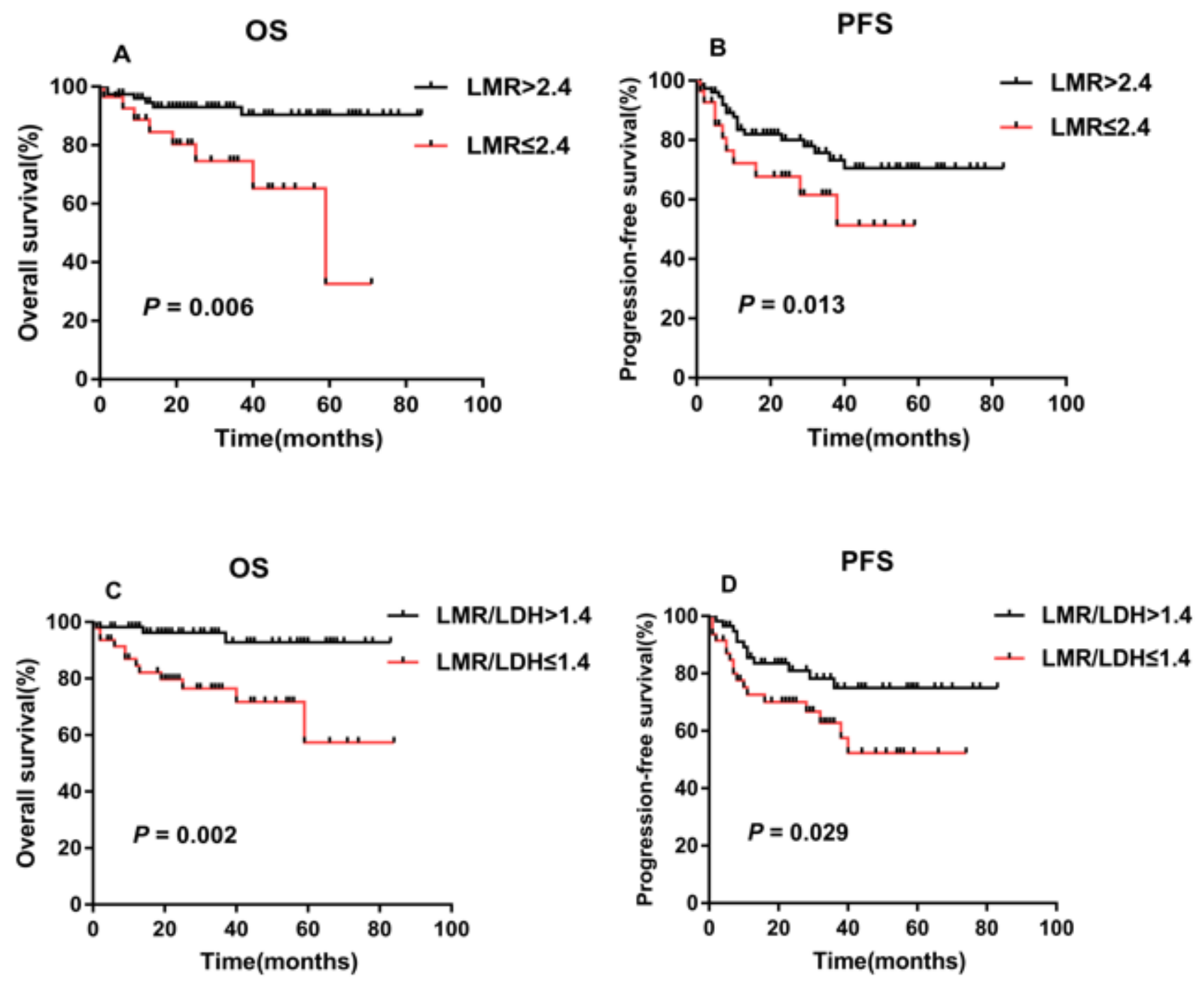

Figure 2

The baseline LMR and LMR/LDH could predict clinical outcome for patients with ALCL. A and B A low baseline LMR was significantly related to poorer OS and PFS (P values: 0.006 and 0.013 , respectively); $C$ and D A low baseline LMR/LDH was significantly associated with shorter OS and PFS (P values: 0.002 and 0.029 , respectively). 

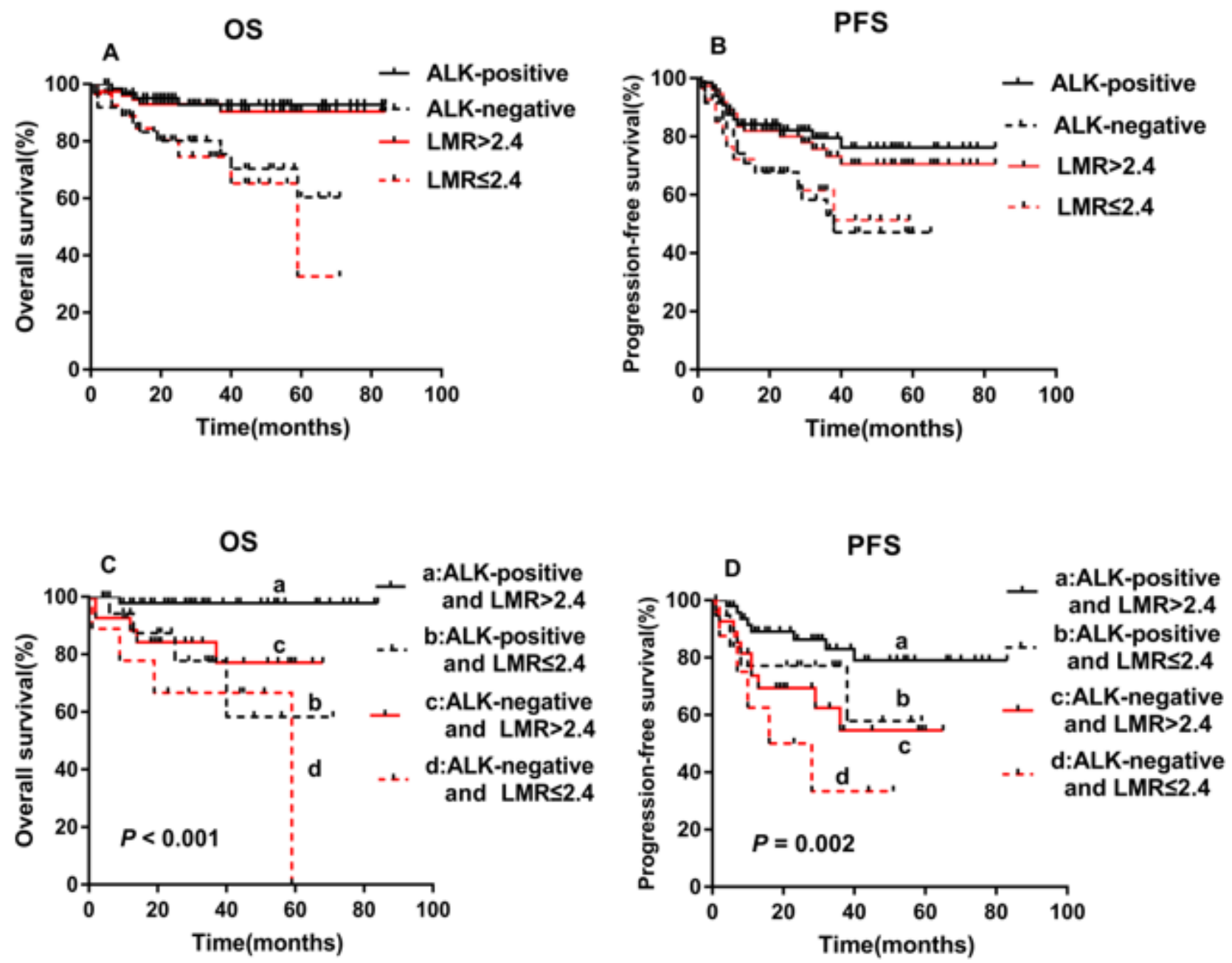

Figure 3

The baseline LMR was highly consistent with ALK expression in predicting prognosis of patients with ALCL. A and B The baseline LMR > 2.4 was similar to ALK positive in OS and PFS of ALCL patients. C and D ALCL patients with a high baseline LMR combined with ALK positive demonstrated better OS and PFS than the other three groups ( $P$ values were $<0.001$ and 0.002 ). 

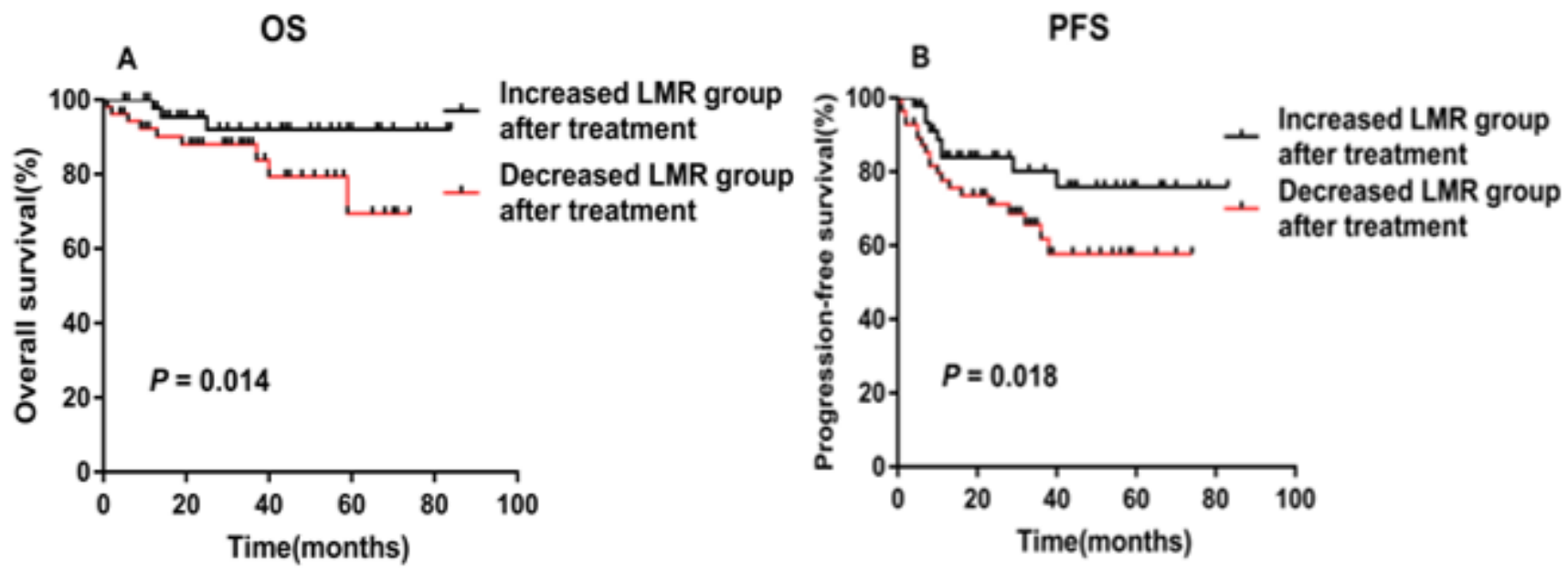

Figure 4

The OS and PFS of patients with an increased LMR after treatment were better than that for patients with a decreased LMR after treatment. A showed that the OS for patients with an increased LMR after treatment was better than that for patients with a decreased LMR after treatment (P value: 0.014). B showed that the PFS for patients with an increased LMR after treatment was better than that for patients with a decreased LMR after treatment (P value: 0.018 ). 

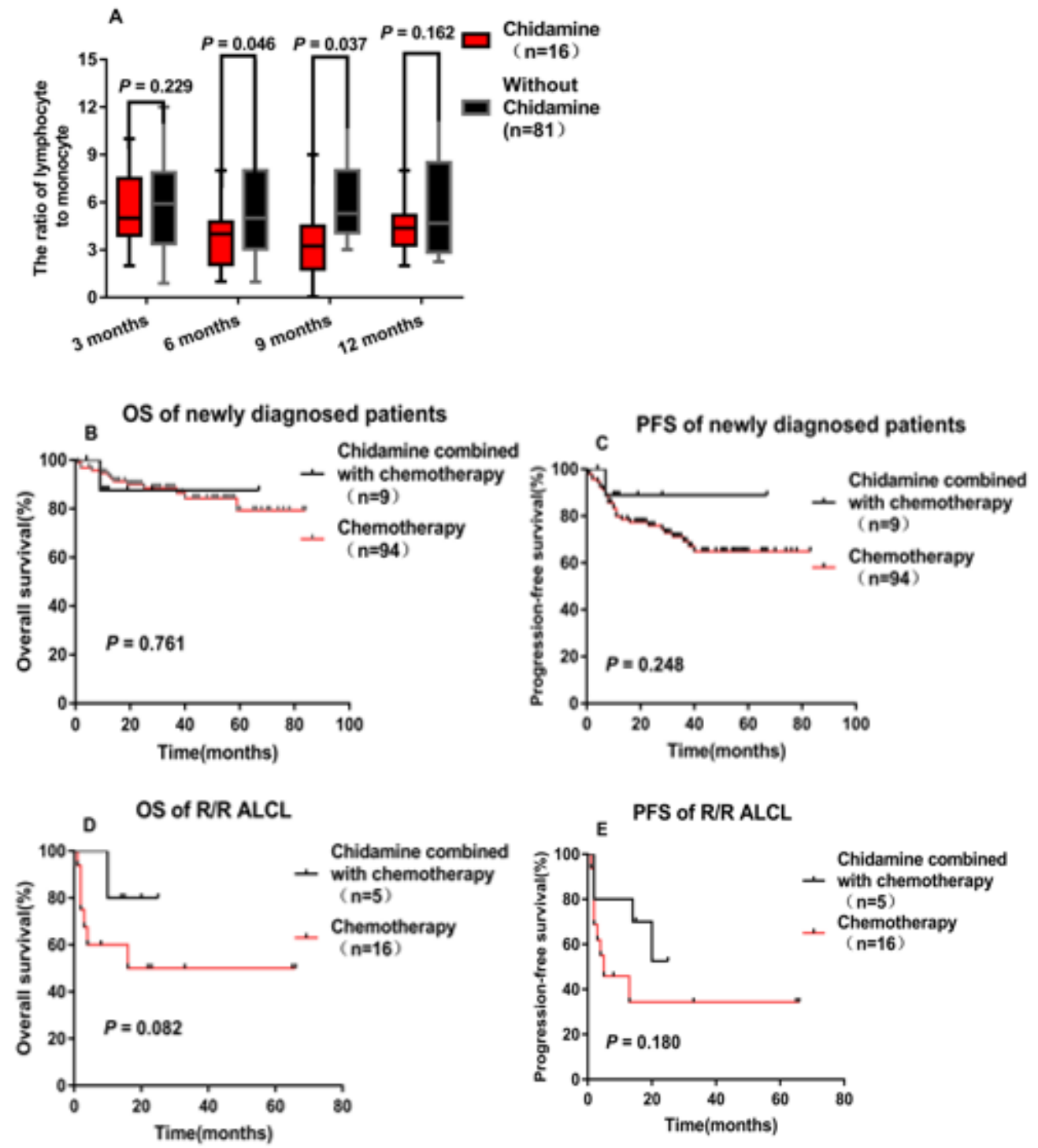

Figure 5

Effects of chidamine treatment on the LMR and survival in patients with ALCL. A showed that the LMR in patients treated with chidamine for 6-9 months was significantly lower than that in patients without chidamine treatment (P values were 0.046 and 0.037 ). Chidamine combined with chemotherapy demonstrated a mild advantage in PFS and OS than chemotherapy alone in newly diagnosed patients (B and $C$ ) and R/R ALCL (D and E).

\section{Supplementary Files}

This is a list of supplementary files associated with this preprint. Click to download.

- Additionalfile1.docx 